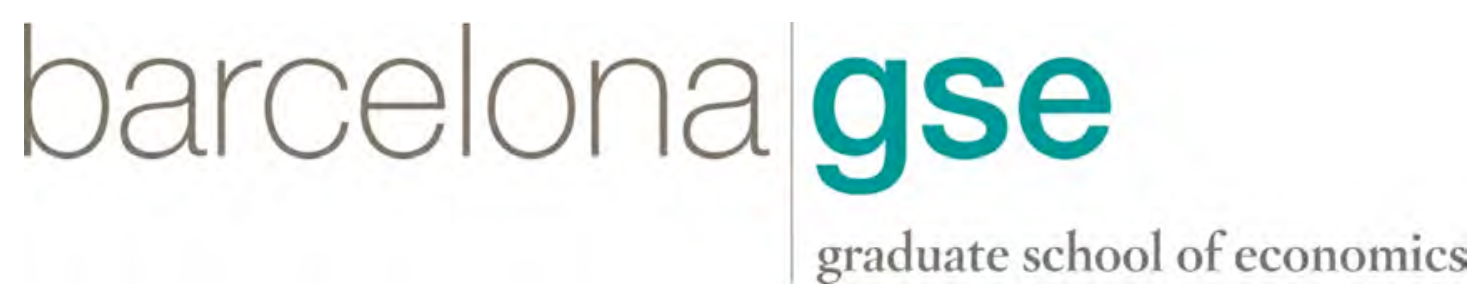

\title{
Monotone Strategyproofness
}

\author{
Hanna Halaburda
}

Guillaume Haeringer

August 2013

Barcelona GSE Working Paper Series

Working Paper $n^{\circ} 712$ 


\title{
Monotone Strategyproofness*
}

\author{
Guillaume Haeringer \\ Universitat Autònoma de Barcelona and Barcelona GSE \\ Hanna Hałaburda \\ Bank of Canada
}

August 7, 2013

\begin{abstract}
We propose a way to compare the extent of preference misrepresentation between two strategies. We define a mechanism to be monotone strategyproof when declaring a "more truthful" preference ordering in the mechanism dominates - with respect to the true preferences - declaring a less truthful preference ordering. Our main result states that a mechanism is strategyproof if, and only if, it is monotone strategyproof. This result holds for any deterministic social choice function on any domain; for probabilistic social choice functions it holds under a mild assumption on the domain.
\end{abstract}

JEL codes $\mathrm{C} 72, \mathrm{D} 41$.

Keywords: strategyproofness, Kemeny sets, misrepresentations, dominant strategy

*We are grateful to Miguel Ángel Ballester, Eric Budish, Lars Ehlers, Matt Jackson, Łukasz Pomorski, Arunava Sen, John Weymark, and especially Vikram Manjunath for their comments and suggestions. Guillaume Haeringer acknowledges the support of Barcelona GSE Research Network and of the Government of Catalonia, Spanish Ministry of Science and Innovation through the grant "Consolidated Group-C" ECO200804756, the Spanish Ministry of Economy and Competitiveness through the Severo Ochoa Programme for Centres of Excellence in R\&D (SEV-2011-0075), and the Generalitat de Catalunya (SGR2009-419). Part of this research was conducted when he was visiting the Stanford Economics department, whose hospitality is gratefully acknowledged. Hanna Hałaburda acknowledges support of HBS Research Division. The views expressed in this paper are those of the authors. No responsibility for them should be attributed to the Bank of Canada. 


\section{INTRODUCTION}

Truthful revelation is a primary goal in mechanism design theory. Ideally, it is a dominant strategy to truthfully reveal one's preferences, and a mechanism that induces such a dominant strategy for all agents and all preference profiles is said to be strategyproof. Such mechanisms do not always exist (Gibbard (1978), Satterthwaite (1978)), but a number of environments have been identified for which they do, e.g. voting, two-sided matching, house allocation, or auctions. ${ }^{1}$

Strategyproof mechanisms induce a radical division between strategies, for they distinguish the truthful strategy from all other strategies. All non-truthful strategies are deemed undesired regardless of their other characteristics - a lie is a lie, whether big or small. This gave the prior literature little reason to scrutinize misrepresentations in strategyproof mechanisms, e.g., measuring how much they deviate from the truth. We argue that this is an important omission and we focus in this paper specifically on non-truthful strategies in strategyproof mechanisms.

There is now growing evidence that strategyproof mechanisms perform poorly in the laboratory (see Chen (2008) for a survey). ${ }^{2}$ Actually, experimental data from games with a dominant strategy also exhibit seemingly irrational behavior. ${ }^{3}$ Overall, most experimental analysis of strategyproof mechanisms cannot go further than acknowledging the percentage of subjects not being truthful and analyzing how this percentage varies when changing some environment parameters or the mechanism itself. However, the existing studies have not been able to rank non-truthful strategies on how close they are to the true preferences, save for some specific cases. This is a serious limitation because one of the valuable attributes of strategyproof mechanisms is their ability to provide quality data about individuals' preferences. Such information is crucial if one wishes to run counter-factuals and test potential new policies. It is easy to imagine that a policy maker (and an econometrician) would prefer a mechanism with a large percentage of individuals not being truthful but "close" to the truth to a mechanism with a smaller percentage of misrepresentations but consisting of large

\footnotetext{
${ }^{1}$ See for instance Moulin (1980) for voting with single-peaked preferences, Dubins and Freedman (1981) and Roth (1982) for two-side matching. See also Barberà (2011) for a recent survey.

${ }^{2}$ See for instance Cason et al. (2006) for the pivotal and the Groves-Clarke mechanisms, Chen and Sönmez (2006) or Calsamiglia et al. (2010) in a matching context.

${ }^{3}$ See Palacios-Hueta and Volij (2009) for the centipede game, Kagel and Levin (1986) for auction games or Andreoni (1995) for public good games.
} 
deviations from the truth.

From a theoretical perspective we argue that studying misrepresentations can help further understand the anatomy of strategyproof mechanisms. By its definition, strategyproofness imposes the existence of a dominant strategy in the mechanism. But does it also impose any structure on misrepresentations? To address this question we classify misrepresentations so as to be able to rank strategies on how much they misrepresent the true preferences. Our contention is that such a classification must be linked to the cost of misrepresenting preferences. In other words - drawing on the intuition for strategyproofness - small misrepresentations should have a lower impact on agents' welfare than large ones, or, put differently, small deviations should dominate large ones. ${ }^{4}$ We call a mechanism satisfying this property monotone strategyproof. One might conjecture that imposing monotonicity between payoffs and distance from the truth would be more restrictive than the usual incentive compatibility, i.e., that some strategyproof mechanisms may not be monotone strategyproof. Our main contribution here is to show that monotone strategyproofness is actually equivalent to strategyproofness. This counterintuitive result turns out to be straightforward to show and holds for a very general class of environments.

Our result is derived within a typical environment where individuals have a preference relation over a finite set of alternatives and participate in a strategyproof mechanism. We first devise a measure to compare the degree of preference misrepresentation. Given two preference orderings $P_{i}$ and $P_{i}^{\prime}$, we define the Kemeny set of $P_{i}$ and $P_{i}^{\prime}$ as the pairs of alternatives that are not ordered in the same way under these two preferences. ${ }^{5}$ We compare the degree of misrepresentation by comparing Kemeny sets: Given a true preference ordering $P_{i}$, ordering $P_{i}^{\prime}$ is defined to be more truthful than $P_{i}^{\prime \prime}$ when the Kemeny set of $P_{i}^{\prime}$ and $P_{i}$ is a subset of that of $P_{i}^{\prime \prime}$ and $P_{i}$. That is, $P_{i}^{\prime}$ is more truthful than $P_{i}^{\prime \prime}$ when $P_{i}^{\prime \prime}$ has relatively more elements whose order disagrees with $P_{i}$. In this context, a mechanism is said to be monotone strategyproof if a more truthful strategy always dominates a less truthful one. ${ }^{6}$ It is straightforward to see that monotone strategyproofness implies strategyproofness. Our main result (Theorem 2) states that the reverse also holds under a mild assumption on the domain of the mechanism. For deterministic social choice function this equivalence holds for

\footnotetext{
${ }^{4}$ See Jackson (2012) for a similar argument in the case of in an exchange economy.

${ }^{5}$ The cardinality of this set is the well-known Kemeny distance (Kemeny, 1959).

${ }^{6}$ The equivalent definition for stochastic mechanisms simply replaces dominance with stochastic dominance.
} 
any environment (Theorem 1).

Given that Kemeny set inclusion captures dominance relations between different strategies in a strategyproof mechanism, it is natural to ask whether the lack of Kemeny set inclusion also implies that there is no dominance; that is, whether the Kemeny set inclusion is equivalent to the dominance. It turns out that this equivalence is true for deterministic mechanisms, but not generally. For non-deterministic mechanisms we show how one preference ordering may dominate another without Kemeny set inclusion. This observation illustrates the complication added by non-deterministic mechanisms.

A paper closely related to ours is Cho (2012), who studies different versions of the concept of stochastic dominance in the context of probabilistic assignment mechanisms. Like us, Cho also presents a result establishing the equivalence between monotone strategyproofness and strategyproofness. His result, proved independently from ours, is however limited to a more specific domain and can be obtained as a corollary of ours. Two other closely related papers are Sato (2013) and Carroll (2012). They also compare "large" and "small" misrepresentations, but they address a different question than we do. Sato and Carroll characterize conditions under which "local" strategyproofness implies "global" strategyproofness. Their concern is more about the transitivity of strategyproofness. Another, somewhat less related paper, is Pathak and Sönmez (2012), who also focus on misrepresentation of preferences. However, Pathak and Sönmez are interested in comparing mechanisms - and therefore consider mechanisms that are not strategyproof - while we are interested in comparing misrepresentations under strategyproof mechanisms.

We outline the environment we consider in Section 2. Monotone strategyproofness is defined and shown to be equivalent to strategyproofness in Section 3. In Section 4 we discuss the relevance of using Kemeny sets to compare strategies and show how similar result can be obtained when agents have cardinal utility functions over outcomes. We conclude in Section 5.

\section{Preliminaries}

Let $N$ be a set of agents and $X$ a (countable) set of alternatives. We shall focus in this paper on the incentives from an individual agent's perspective, henceforth called agent $i{ }^{7} \mathrm{~A}$

\footnotetext{
${ }^{7}$ Thus, the set of individuals need not be finite nor countable.
} 
preference $P_{i}$ for agent $i$ over $X$ is a linear order on $X$. Given a preference relation $P_{i}$ we denote by $R_{i}$ the weak ordering associated to $P_{i}$, i.e., $x R_{i} x^{\prime}$ implies $x P_{i} x^{\prime}$ or $x=x^{\prime} .{ }^{8}$

A preference profile is a list $P$ of preferences for each agent $j \in N, P=\times_{j \in N} P_{j}$. We follow the usual convention to denote by $P_{-i}$ the profile $\left(P_{1}, \ldots, P_{i-1}, P_{i+1}, \ldots, P_{n}\right)$. The set of all possible preferences for agent $i$ is denoted $\mathcal{P}_{i}$ and the set of all possible preference profiles, called the universal domain, is $\mathcal{P}$. A domain is a non-empty subset of $\mathcal{P}$.

Two preference orderings $P_{i}$ and $P_{i}^{\prime}$ are adjacent if there exists a pair of alternatives $\left(x, x^{\prime}\right)$ such that $x P_{i} x^{\prime}, x^{\prime} P_{i}^{\prime} x$, and for each pair of alternatives $\left(z, z^{\prime}\right) \neq\left(x, x^{\prime}\right), z P_{i} z^{\prime} \Leftrightarrow z P_{i}^{\prime} z^{\prime}$. A domain $\mathcal{D}$ is connected if for any two preference orderings $P_{i}, P_{i}^{\prime} \in \mathcal{D}$, there exists a sequence $\left(P^{1}, \ldots, P^{\ell}\right)$ such that $P^{1}=P_{i}, P^{\ell}=P_{i}^{\prime}$ and for each $h<l, P^{h}$ and $P^{h+1}$ are adjacent. A domain $\mathcal{D}$ is strongly connected if it is connected and there is a sequence of adjacent preference orderings $\left(P^{1}, \ldots, P^{\ell}\right)$ such that $P^{1}=P_{i}, P^{\ell}=P_{i}^{\prime}$ and for each $h<l$, if for some $x, x^{\prime} \in X$ we have $x P^{h} x^{\prime}$ and $x^{\prime} P^{h+1} x$, then for each $h^{\prime}>h$ we have $x^{\prime} P^{h^{\prime}} x .^{9}$ Sato (2013) shows that the single-peaked domain is strongly connected and Carroll (2012) shows that the (maximal) single-crossing domain is also strongly connected. ${ }^{10}$

A lottery is a vector of probabilities $\pi \in \mathbb{R}^{|X|}$ such that $\sum_{x \in X} \pi_{x}=1$. We denote by $\Delta(X)$ the set of all lotteries over $X$.

A social choice function (or a mechanism) on a domain $\mathcal{D}$ is a mapping $\varphi: \mathcal{D} \rightarrow$ $\Delta(X)$. Given a profile $P$, we denote by $\varphi_{x}(P)$ the probability of alternative $x$ under the lottery $\varphi(P)$. The social choice function is deterministic if for each $P \in \mathcal{D}, \varphi(P)$ is a degenerate lottery. In this case (abusing notation) we shall denote by $\varphi\left(P_{i}, P_{-i}\right)$ the alternative $x$ such that $\varphi_{x}\left(P_{i}, P_{-i}\right)=1$.

Given preference orderings $P_{i}, P_{i}^{\prime}, P_{i}^{\prime \prime}$, we say that $P_{i}^{\prime}$ (stochastically) dominates $P_{i}^{\prime \prime}$

\footnotetext{
${ }^{8}$ Note that in this setting agent $i$ 's preferences are over the set of outcomes, which may not be always the case. In the two-sided matching literature for instance, agents are defined as having preferences over potential partners while an outcome is a matching that involves all agents. Since our model only describes the problem from a unique agent's perspective this is without loss of generality.

${ }^{9}$ Sato (2013) calls a strongly connected domain a connected domain that satisfies the non-restoration property. The strongly connected domain defined by Chatterji et al. rests on a similar notion but the notion of connectedness is imposed on alternatives and not preferences.

${ }^{10}$ Both single-peakedness and single-crossingness assume the existence of an ordering of alternatives, and admissible preferences are obtained using this ordering. It is of course possible to have a (small) domain that satisfies the properties required by single-peakedness or single-crossingness but does not contain enough preferences such that any pair of preferences in the domain are connected.
} 
with respect to $P_{i}$, denoted $P_{i}^{\prime} \gg^{P_{i}} P_{i}^{\prime \prime}$, when

for each $P_{-i}$, for each $x \in X, \quad \sum_{x^{\prime}: x^{\prime} R_{i} x} \varphi_{x^{\prime}}\left(P_{i}^{\prime}, P_{-i}\right) \geq \sum_{x^{\prime}: x^{\prime} R_{i} x} \varphi_{x^{\prime}}\left(P_{i}^{\prime \prime}, P_{-i}\right)$.

For a deterministic social choice function condition (1) can be rewritten as

$$
\text { for each } P_{-i}, \quad \varphi\left(P_{i}, P_{-i}\right) R_{i} \varphi\left(P_{i}^{\prime}, P_{-i}\right)
$$

Definition 1 A social function $\varphi$ is strategyproof on a domain $\mathcal{D}$ if for each agent $i \in N$, and for each $P_{i}, P_{i}^{\prime} \in \mathcal{D}, P_{i}$ dominates $P_{i}^{\prime}$ with respect to $P_{i}$.

Observe that the sets of individuals, the (true) preference profile $P$, and a social choice function $\varphi$ on a domain $\mathcal{D}$ induce a strategic form game $\Gamma^{\varphi}=\langle N, \mathcal{D}, P\rangle$, where $N$ is the set of players, $P$ is the set of (pure) strategy profiles, the outcome of a strategy profile $P$ is given by $\varphi(P)$, and each player $i \in N$ evaluates the outcome $\varphi(P)$ using his true preferences $P_{i}$. In this context, a social choice function $\varphi$ is strategyproof if in the game form $\Gamma^{\varphi}$ induced by $\varphi$ the true strategy $P_{i}$ is a (weakly) dominant strategy for each player $i$.

\section{Monotone STRATEGyProOfness}

One natural way to compare two preference orderings is by counting the number of pairs of alternatives whose relative rank differ between the two orderings. This method is known as the Kemeny distance (Kemeny, 1959). However, it is obvious to see that this method serves little for our purposes. Indeed, the Kemeny distance produces a complete (weak) ordering of strategies, while the ordering produced by the concept of strategic dominance is not guaranteed to be complete. One way to keep an incomplete relation when comparing strategies is to consider what we call the Kemeny sets of the preference orderings.

Definition 2 Given two preference orderings $P_{i}, P_{i}^{\prime}$, the Kemeny set of $P_{i}$ and $P_{i}^{\prime}$ is the set of all pairs $\left(x, x^{\prime}\right) \in X \times X$ that are not ordered identically in $P_{i}$ and $P_{i}^{\prime}$,

$$
K\left(P_{i}, P_{i}^{\prime}\right)=\left\{\left(x, x^{\prime}\right) \in X \times X: x^{\prime} P_{i} x \text { and } x P_{i}^{\prime} x^{\prime}\right\}
$$

We are now ready to introduce our main concept: 
Definition 3 A social choice function is monotone strategyproof on a domain $\mathcal{D}$ if for each $P_{i} \in \mathcal{D}$ and each pair $P_{i}^{\prime}, P_{i}^{\prime \prime}$ such that $K\left(P_{i}^{\prime}, P_{i}\right) \subset K\left(P_{i}^{\prime \prime}, P_{i}\right), P_{i}^{\prime}$ dominates $P_{i}^{\prime \prime}$ with respect to $P_{i}$.

Note that if a social choice function is monotone strategyproof it is obviously strategyproof. Since $K\left(P_{i}, P_{i}\right)=\emptyset, P_{i}$ dominates any other preference ordering $P_{i}^{\prime}$. In following theorem we show that the converse also holds for deterministic social functions on any domain.

Theorem 1 Let $\varphi$ be a deterministic social choice function on a domain $\mathcal{D}$. Then $\varphi$ is strategyproof if, and only if, it is monotone strategyproof.

Proof Let $P_{i}, P_{i}^{\prime}$ and $P_{i}^{\prime \prime}$ such that $K\left(P_{i}\right) \subset K\left(P_{i}^{\prime}\right)$ with $P_{i}, P_{i}^{\prime}, P_{i}^{\prime \prime} \in \mathcal{D}$. Let $P_{-i}$ by any profile, and let $x^{\prime}=\varphi\left(P_{i}^{\prime}, P_{-i}\right)$ and $x^{\prime \prime}=\varphi\left(P_{i}^{\prime \prime}, P_{-i}\right)$ and assume that $x^{\prime} \neq x^{\prime \prime}{ }^{11}$

Observe that if $x^{\prime} P_{i}^{\prime \prime} x^{\prime \prime}$, then $\varphi$ cannot be strategyproof. This is because if $P_{i}^{\prime \prime}$ where the true preferences, then individual $i$ can benefit by reporting $P_{i}^{\prime}$ instead of $P_{i}^{\prime \prime}$. Similarly, it cannot be that $x^{\prime \prime} P_{i}^{\prime} x^{\prime}$. So it must be that $x^{\prime} P_{i}^{\prime} x^{\prime \prime}$ and $x^{\prime \prime} P_{i}^{\prime \prime} x^{\prime}$. Since $K\left(P_{i}^{\prime}, P_{i}\right) \subset K\left(P_{i}^{\prime \prime}, P_{i}\right)$, we have $x^{\prime} P_{i} x^{\prime \prime}$. That is, $\varphi\left(P_{i}^{\prime}, P_{-i}\right) P_{i} \varphi\left(P_{i}^{\prime \prime}, P_{-i}\right)$.

A similar result holds when we do not require the social choice function to be deterministic, provided some restrictions on the domain.

Proposition 1 Let $\varphi$ be a social choice function on a strongly connected domain $\mathcal{D}$. Then $\varphi$ is strategyproof if, and only if, it is monotone strategyproof.

Proof Let $P_{i}=x_{1}, x_{2}, \ldots x_{\ell}$, and let $P_{i}^{\prime}$ and $P_{i}^{\prime \prime}$ such that $K\left(P_{i}^{\prime}, P_{i}\right) \subset K\left(P_{i}^{\prime \prime}, P_{i}\right)$. Since the domain is strongly connected, there exist $P_{i}^{1}, \ldots, P_{i}^{\ell}$ such that $P_{i}^{\prime}=P_{i}^{1}, P_{i}^{\prime \prime}=P_{i}^{\ell}$, and for each $h<\ell, K\left(P_{i}^{h}, P_{i}\right) \subset K\left(P_{i}^{h+1}, P_{i}\right)$, with $P_{i}^{h}$ and $P_{i}^{h+1}$ being adjacent. Since the stochastic dominance relation is transitive, it is sufficient to show that for any $h \leq \ell$ we have $P_{i}^{h} \gg^{P_{i}} P_{i}^{h+1}$.

Consider then any $h \leq \ell$, and let $x, x^{\prime}$ be the pair of alternatives such that $x P_{i}^{h} x^{\prime}$ and $x^{\prime} P_{i}^{h+1} x$. Let $Z=\left\{x^{\prime \prime}: x^{\prime \prime} P_{i}^{h} x\right\} \equiv\left\{x^{\prime \prime}: x^{\prime \prime} P_{i}^{h+1} x^{\prime}\right\}$. Observe that restricted on $Z, P_{i}^{h}$ and $P_{i}^{h+1}$ are identical. By strategyproofness, $P^{h} \gg^{P^{h}} P^{h+1}$, and $P^{h+1} \gg^{P^{h+1}} P^{h}$. So for any

\footnotetext{
${ }^{11}$ If $\varphi\left(P_{i}^{\prime}, P_{-i}\right)=\varphi\left(P_{i}^{\prime \prime}, P_{-i}\right)$ for any profile $P_{-i}$, then $P_{i}^{\prime}$ and $P_{i}^{\prime \prime}$ are equivalent strategies and thus $P_{i}^{\prime}$ trivially dominates $P_{i}^{\prime \prime}$.
} 
$P_{-i}$ and each $x^{\prime \prime} \in Z$ we have

$$
\sum_{\hat{x} R_{i}^{h} x^{\prime \prime}} \varphi_{\hat{x}}\left(P_{i}^{h}, P_{-i}\right) \geq \sum_{\hat{x} R_{i}^{h} x^{\prime \prime}} \varphi_{\hat{x}}\left(P_{i}^{h+1}, P_{-i}\right) \quad \text { and } \sum_{\hat{x} R_{i}^{h} x^{\prime \prime}} \varphi_{\hat{x}}\left(P_{i}^{h+1}, P_{-i}\right) \geq \sum_{\hat{x} R_{i}^{h} x^{\prime \prime}} \varphi_{\hat{x}}\left(P_{i}^{h}, P_{-i}\right) .
$$

So, for each $x^{\prime \prime} \in Z$ we have $\varphi_{x^{\prime \prime}}\left(P_{i}^{h+1}, P_{-i}\right)=\varphi_{x^{\prime \prime}}\left(P_{i}^{h}, P_{-i}\right)$. We easily obtain a similar result for $\hat{Z}=\left\{x^{\prime \prime}: x^{\prime} P_{i}^{h} x^{\prime \prime}\right\} \equiv\left\{x^{\prime \prime}: x P_{i}^{h+1} x^{\prime \prime}\right\} \equiv X \backslash\left(Z \cup\left\{x, x^{\prime}\right\}\right)$. So, for any $P_{-i}$,

$$
\varphi_{\hat{x}}\left(P_{i}^{h+1}, P_{-i}\right)=\varphi_{\hat{x}}\left(P_{i}^{h}, P_{-i}\right) \quad \text { for each } \hat{x} \in X \backslash\left\{x, x^{\prime}\right\} .
$$

It follows that $P^{h} \gg^{P^{h}} P^{h+1}$ and $P^{h+1} \gg^{P^{h+1}} P^{h}$ imply

$$
\begin{aligned}
\varphi_{x}\left(P^{h}, P_{-i}\right) & \geq \varphi_{x}\left(P^{h+1}, P_{-i}\right) \\
\varphi_{x}\left(P^{h}, P_{-i}\right)+\varphi_{x^{\prime}}\left(P^{h}, P_{-i}\right) & =\varphi_{x}\left(P^{h+1}, P_{-i}\right)+\varphi_{x^{\prime}}\left(P^{h+1}, P_{-i}\right) .
\end{aligned}
$$

Since $\left(x, x^{\prime}\right) \notin K\left(P_{i}^{h}, P_{i}\right)$ and $\left(x, x^{\prime}\right) \in K\left(P_{i}^{h+1}, P_{i}\right), x P_{i} x^{\prime}$. Together (4), (5) and (6) imply that for each $\hat{x} \in X$ and any $P_{-i}$,

$$
\sum_{x^{\prime \prime} R_{i} \hat{x}} \varphi_{x^{\prime \prime}}\left(P^{h}, P_{-i}\right) \geq \sum_{x^{\prime \prime} R_{i} \hat{x}} \varphi_{x^{\prime \prime}}\left(P^{h+1}, P_{-i}\right) .
$$

That is, $P^{h} \gg^{P_{i}} P^{h+1}$, the desired result.

The next lemma shows that monotone strategyproofness may imply strategyproofness also for some domains that are not strongly connected.

Lemma 1 Let $P_{i}$ and $P_{i}^{\prime \prime}$ be such that for some distinct alternatives $x_{1}$ and $x_{k}$ it holds that $\left(x, x^{\prime}\right) \in K\left(P_{i}, P_{i}^{\prime \prime}\right)$ if, and only if, $x_{1} R_{i} x P_{i} x^{\prime} R_{i} x_{k}$ and $x_{k} R_{i}^{\prime \prime} x P_{i}^{\prime \prime} x^{\prime} R_{i}^{\prime \prime} x_{1}{ }^{12}$ Let $\varphi$ be any stochastic strategyproof mechanism. If $K\left(P_{i}^{\prime}, P_{i}\right) \subset K\left(P_{i}^{\prime \prime}, P_{i}\right)$, then $P_{i}^{\prime} \gg^{P_{i}} P_{i}^{\prime \prime}$.

Proof Let $P_{i}=x_{1}, \ldots, x_{k}$ and assume without loss of generality that $P_{i}^{\prime \prime}=x_{k}, \ldots, x_{1} \cdot{ }^{13}$ Let $P_{i}^{\prime}$ such that $K\left(P_{i}, P_{i}^{\prime}\right) \subset K\left(P_{i}, P_{i}^{\prime \prime}\right)$. Consider any profile $P_{-i}$ and let $\pi_{h}, \pi_{h}^{\prime}$ and $\pi_{h}^{\prime \prime}$

\footnotetext{
${ }^{12}$ That is, $P_{i}^{\prime \prime}$ is a complete reversal of $P_{i}$ when restricting both preferences to the alternatives that lie between $x_{1}$ and $x_{k}$ in $P_{i}$.

${ }^{13}$ If there are some alternatives $x_{1}^{\prime}, \ldots, x_{\ell}^{\prime}$ such that $P_{i}=x_{1}^{\prime}, \ldots, x_{\ell}^{\prime}, x_{1}, \ldots x_{k}, \ldots$ then $P_{i}^{\prime \prime}=$ $x_{1}^{\prime}, \ldots, x_{\ell}^{\prime}, x_{k}, \ldots, x_{1}, \ldots$ and thus the first $\ell$ alternatives for any $P_{i}^{\prime}$ such that $K\left(P_{i}, P_{i}^{\prime}\right) \subset K\left(P_{i}, P_{i}^{\prime \prime}\right)$ are, in this order, $x_{1}^{\prime}, \ldots, x_{\ell}^{\prime}$. In this case, for any profile $P_{-i}$ and strategyproof mechanism $\varphi$ we have $\varphi_{x_{h}^{\prime}}\left(P_{i}, P_{-i}\right)=\varphi_{x_{h}^{\prime}}\left(P_{i}^{\prime}, P_{-i}\right)=\varphi_{x_{h}^{\prime}}\left(P_{i}^{\prime \prime}, P_{-i}\right)$, for $h=1, \ldots, \ell$.
} 
denote the probability of alternative $x_{h}$ under the strategy $P_{i}, P_{i}^{\prime}$ and $P_{i}^{\prime \prime}$, respectively. We need to show that $P_{i} \gg^{P_{i}} P_{i}^{\prime \prime}$, that is,

$$
\begin{aligned}
\pi_{1}^{\prime} & \geq \pi_{1}^{\prime \prime} \\
\pi_{1}^{\prime}+\pi_{2}^{\prime} & \geq \pi_{1}^{\prime \prime}+\pi_{2}^{\prime \prime} \\
\cdots & \\
\pi_{1}^{\prime}+\cdots+\pi_{k-1}^{\prime} & \geq \pi_{1}^{\prime \prime}+\cdots+\pi_{k-1}^{\prime \prime} \\
\pi_{1}^{\prime}+\cdots+\pi_{k-1}^{\prime}+\pi_{k}^{\prime} & \geq \pi_{1}^{\prime \prime}+\cdots+\pi_{k-1}^{\prime \prime}+\pi_{k}^{\prime \prime}
\end{aligned}
$$

Strategyproofness of $\varphi$ implies

$$
\begin{aligned}
& \pi_{k}^{\prime \prime} \geq \pi_{k}^{\prime} \\
& \pi_{k}^{\prime \prime}+\pi_{k-1}^{\prime \prime} \geq \pi_{k}^{\prime}+\pi_{k-1}^{\prime} \\
& \cdots \\
& \pi_{k}^{\prime \prime}+\cdots+\pi_{2}^{\prime \prime} \geq \pi_{k}^{\prime}+\cdots+\pi_{2}^{\prime} \\
& \pi_{k}^{\prime \prime}+\cdots+\pi_{2}^{\prime \prime}+\pi_{1}^{\prime \prime} \geq \pi_{k}^{\prime}+\cdots+\pi_{2}^{\prime}+\pi_{1}^{\prime}
\end{aligned}
$$

Since there are $k$ alternatives $\sum_{h} \pi_{h}^{\prime}=\sum_{h} \pi_{h}^{\prime \prime}=1$. It is then readily verified that $\left(a_{k}^{\prime}\right)$, $\left(a_{k-1}^{\prime}\right), \ldots,\left(a_{2}^{\prime}\right)$ imply $\left(a_{k-1}\right),\left(a_{k-2}\right), \ldots,\left(a_{1}\right)$, respectively.

Building on the intuition of Proposition 1 and Lemma 1 we can identify a larger class of domains where the equivalence between strategy proofness and monotone strategyproofness holds. For this purpose, it is useful to introduce the concept of tight domain.

Let $P_{i}^{\prime}$ and $P_{i}^{\prime \prime}$ be any pair of preference orderings, and let $G\left(P_{i}^{\prime}, P_{i}^{\prime \prime}\right)=\left(X, K\left(P_{i}^{\prime}, P_{i}^{\prime \prime}\right)\right)$ be a graph where $X$ is the set of vertices and $K\left(P_{i}^{\prime}, P_{i}^{\prime \prime}\right)$ is the set of edges. For instance, if $K\left(P_{i}^{\prime}, P_{i}^{\prime \prime}\right)=\left\{\left(x_{1}, x_{2}\right),\left(x_{2}, x_{4}\right),\left(x_{3}, x_{5}\right)\right\}$, then the graph $G\left(P_{i}^{\prime}, P_{i}^{\prime \prime}\right)$ has three edges: between $x_{1}$ and $x_{2}$, between $x_{2}$ and $x_{4}$, and between $x_{3}$ and $x_{5}$.

Given a graph $G\left(P_{i}^{\prime}, P_{i}^{\prime \prime}\right)$, a path is an ordered sequence of alternatives $\left(x_{1}, \ldots, x_{k}\right)$ such that for each $h<k,\left(x_{h}, x_{h+1}\right) \in K\left(P_{i}^{\prime}, P_{i}^{\prime \prime}\right)$. Two alternatives $x$ and $x^{\prime}$ are connected if there exists a path $\left(x_{1}, \ldots, x_{k}\right)$ with $x=x_{1}$ and $x^{\prime}=x_{k}$. A connected component $X^{\prime} \subseteq X$ is a set of alternatives such that any two alternatives in $X^{\prime}$ are connected in $G\left(P_{i}^{\prime}, P_{i}^{\prime \prime}\right)$ and no alternative in $X^{\prime}$ is connected with an alternative in $X \backslash X^{\prime}$. Finally, a Kemeny set $K\left(P_{i}^{\prime}, P_{i}^{\prime \prime}\right)$ is thin if the largest connected component in the graph $G\left(P_{i}^{\prime}, P_{i}^{\prime \prime}\right)$ is of size 3 or less. 
A domain $\mathcal{D}$ is tight if for any pair of preferences $\left(P_{i}^{\prime}, P_{i}^{\prime \prime}\right)$ there exists a sequence of preference orderings $\left(P^{1}, \ldots, P^{\ell}\right)$ such that $P^{1}=P_{i}, P^{\ell}=P_{i}^{\prime}$ and for any $k=1, \ldots, l-1$ the Kemeny set $K\left(P^{k}, P^{k+1}\right)$ is thin.

Theorem 2 Let $\varphi$ be a social choice function on a tight domain $\mathcal{D}$. Then $\varphi$ is strategyproof if, and only if, it is monotone strategyproof.

The proof of Theorem 2 follows similar logic as the proof of Proposition 1.

Proof Consider a tight domain. Clearly, it suffices to show that strategyproofness implies monotone strategyproofness. Let $P_{i}=x_{1}, x_{2}, \ldots x_{\ell}$, and let $P_{i}^{\prime}$ and $P_{i}^{\prime \prime}$ such that $K\left(P_{i}^{\prime}, P_{i}\right) \subset$ $K\left(P_{i}^{\prime \prime}, P_{i}\right)$. Since the domain is tight, there exists sequence $\left(P^{1}, \ldots, P^{\ell}\right)$ such that $P^{1}=P_{i}^{\prime}$, $P^{\ell}=P_{i}^{\prime \prime}$ and for any $k=1, \ldots, l-1$ the Kemeny set $K\left(P^{h}, P^{h+1}\right)$ is thin. Since the stochastic dominance relation is transitive, it is sufficient to show that for any $h \leq \ell$ we have $P^{h} \gg^{P_{i}} P^{h+1}$.

In the first step, we show that for any connected component in the graph representing $K\left(P^{h}, P^{h+1}\right)$ the sum of probabilities for getting better alternatives than the nodes of the component are equal. Let a set of alternatives $\mathcal{C} \subset X$ be any connected component in the graph representing $K\left(P^{h}, P^{h+1}\right)$. Let $\min (\mathcal{C}, P)$ be the least preferred element of $\mathcal{C}$, i.e.,

$$
\min (\mathcal{C}, P)=x \Longleftrightarrow x \in \mathcal{C} \quad \& \quad \forall x^{\prime} \in \mathcal{C}, x P x^{\prime}
$$

Similarly, let $\max (\mathcal{C}, P)$ be the most preferred element.

By strategy-proofness:

$$
\text { and } \begin{aligned}
\sum_{x: x P^{h} \max (\mathcal{C}, P)} \pi_{x}^{h} & \geq \sum_{x: x P^{h} \max (\mathcal{C}, P)} \pi_{x}^{h+1} \\
\sum_{x: x P^{h+1} \max (\mathcal{C}, P)} \pi_{x}^{h+1} & \geq \sum_{x: x P^{h+1} \max (\mathcal{C}, P)} \pi_{x}^{h} .
\end{aligned}
$$

Notice, however that $\left\{x: x P^{h} \max (\mathcal{C}, P)\right\}=\left\{x: x P^{h+1} \max (\mathcal{C}, P)\right\}=\mathcal{A}$. Assume, to the contrary, that there exists $y$ such that $y P^{h+1} \max (\mathcal{C}, P)$ but $\max (\mathcal{C}, P) P^{h} y$. But then $y \in \mathcal{C}$, and there would be no $P$ such that $y P^{h+1} \max (\mathcal{C}, P)$. Thus, conditions (8) and (9) can be rewritten

$$
\sum_{\mathcal{A}} \pi_{x}^{h} \geq \sum_{\mathcal{A}} \pi_{x}^{h+1} \quad \& \quad \sum_{\mathcal{A}} \pi_{x}^{h+1} \geq \sum_{\mathcal{A}} \pi_{x}^{h} \Longrightarrow \sum_{\mathcal{A}} \pi_{x}^{h}=\sum_{\mathcal{A}} \pi_{x}^{h+1}
$$


Moreover, similarly we can show that $\sum_{\mathcal{A} \cup \mathcal{C}} \pi_{x}^{h}=\sum_{\mathcal{A} \cup \mathcal{C}} \pi_{x}^{h+1}$, which implies that

$$
\sum_{\mathcal{C}} \pi_{x}^{h}=\sum_{\mathcal{C}} \pi_{x}^{h+1}
$$

A connected component in $K\left(P^{h}, P^{h+1}\right)$ may have either two or three nodes. First, consider the case where the connected component has two nodes, $|\mathcal{C}|=2$. Let $a=\max \left(\mathcal{C}, P_{i}\right)$ and $b=\min \left(\mathcal{C}, P_{i}\right)$. Because (with slight abuse of notation) $K\left(P^{h}, P_{i}\right) \cap \mathcal{C} \subset K\left(P^{h+1}, P_{i}\right) \cap \mathcal{C}$, it must be that $a P^{h} b$ and $b P^{h+1} a$. Then by strategy-proofness $\sum_{x: x R^{h+1} b} \pi_{x}^{h+1} \geq \sum_{x: x R^{h+1 b}} \pi_{x}^{h}$; and together with (10) it yields $\pi_{b}^{h+1} \geq \pi_{b}^{h}$. Equality (11) implies $\pi_{a}^{h+1}+\pi_{b}^{h+1}=\pi_{a}^{h}+\pi_{b}^{h}$. Thus,

$$
\pi_{a}^{h} \geq \pi_{a}^{h+1}
$$

and $\quad \pi_{a}^{h}+\pi_{b}^{h}=\pi_{a}^{h+1}+\pi_{b}^{h+1}$.

Next, consider the case where the connected component has three nodes, $|\mathcal{C}|=3$. Let $a=\max \left(\mathcal{C}, P_{i}\right), c=\min \left(\mathcal{C}, P_{i}\right)$ and $b$ is such that $a P_{i} b$ and $b P_{i} a$. Let $y=\max \left(\mathcal{C}, P^{h+1}\right)$, $y^{\prime \prime}=\min \left(\mathcal{C}, P^{h+1}\right)$ and $y^{\prime}$ be such that $y P^{h+1} y^{\prime}$ and $y^{\prime} P^{h+1} y^{\prime \prime}$. Then, by strategy-proofness and equality (10):

$$
\begin{aligned}
\pi_{y}^{h+1} & \geq \pi_{y}^{h} \\
\pi_{y}^{h+1}+\pi_{y^{\prime}}^{h+1} & \geq \pi_{y}^{h}+\pi_{y^{\prime}}^{h} \\
\pi_{y}^{h+1}+\pi_{y^{\prime}}^{h+1}+\pi_{y^{\prime \prime}}^{h+1} & =\pi_{y}^{h}+\pi_{y^{\prime}}^{h}+\pi_{y^{\prime \prime}}^{h} .
\end{aligned}
$$

Moreover, $K\left(P^{h}, P^{*}\right) \cap \mathcal{C} \subset K\left(P^{h+1}, P^{*}\right) \cap \mathcal{C}$. Then, by Lemma 1 we obtain

$$
\begin{aligned}
\pi_{a}^{h} & \geq \pi_{a}^{h+1} \\
\pi_{a}^{h}+\pi_{b}^{h} & \geq \pi_{a}^{h+1}+\pi_{b}^{h+1} \\
\pi_{a}^{h}+\pi_{b}^{h}+\pi_{c}^{h} & =\pi_{a}^{h+1}+\pi_{b}^{h+1}+\pi_{c}^{h+1} .
\end{aligned}
$$

Lastly, notice that for alternatives (again, with slight abuse of notation) $z \notin K\left(P^{h}, P^{h+1}\right)$, $\pi_{z}^{h}=\pi_{z}^{h+1}$. Therefore, for any $x$

$$
\sum_{x^{\prime}: x^{\prime} R^{*} x} \pi_{x^{\prime}}^{h} \geq \sum_{x^{\prime}: x^{\prime} R^{*} x} \pi_{x^{\prime}}^{h+1}
$$

i.e., $P^{h}$ stochastically dominated $P^{h+1}$ with respect to $P_{i}$. 
A tightness of the domain is a sufficient condition to obtain the equivalence of strategyproofness and monotone strategyproofness, but - similarly to strong connectedness - it is not a necessary condition. The following two examples show that the equivalence may fail (Example 1) or hold (Example 2) when the domain is not tight.

Example 1 Let $X=\left\{x_{1}, x_{2}, x_{3}, x_{4}\right\}$, and let $\mathcal{D}$ be the domain composed of the three preference orderings depicted in Table 1 . Preferences $P_{i}, P_{i}^{\prime}$ and $P_{i}^{\prime \prime}$ satisfy $K\left(P_{i}^{\prime}, P_{i}\right) \subset K\left(P_{i}^{\prime \prime}, P_{i}\right)$.

\begin{tabular}{ccc}
\hline$P_{i}$ & $P_{i}^{\prime}$ & $P_{i}^{\prime \prime}$ \\
\hline \hline$x_{1}$ & $x_{1}$ & $x_{3}$ \\
$x_{2}$ & $x_{4}$ & $x_{4}$ \\
$x_{3}$ & $x_{2}$ & $x_{2}$ \\
$x_{4}$ & $x_{3}$ & $x_{1}$ \\
\hline
\end{tabular}

Table 1: A non-tight domain

Suppose that for any $P_{-i}$ the probability to obtain alternative $x \in X$ is given by the Table 2. One easily verifies that $\hat{P}_{i} \gg \hat{P}_{i} \tilde{P}_{i}$ for any $\hat{P}_{i}, \tilde{P}_{i} \in\left\{P_{i}, P_{i}^{\prime}, P_{i}^{\prime \prime}\right\}$, i.e., strategyproof-

\begin{tabular}{cccc}
\hline & $P_{i}$ & $P_{i}^{\prime}$ & $P_{i}^{\prime \prime}$ \\
\hline \hline$x_{1}$ & .51 & .51 & .07 \\
$x_{2}$ & .3 & .05 & .33 \\
$x_{3}$ & .18 & .004 & .4 \\
$x_{4}$ & .01 & .4 & .2 \\
\hline
\end{tabular}

Table 2: Probabilities of each alternative under $P_{i}, P_{i}^{\prime}$ and $P_{i}^{\prime \prime}$

ness holds in this example. However, in spite of having $K\left(P_{i}^{\prime}, P_{i}\right) \subset K\left(P_{i}^{\prime \prime}, P_{i}\right)$ it is not the case that $P_{i}^{\prime}$ dominates $P_{i}^{\prime \prime}$ with respect to $P_{i}$. Indeed, $\sum_{x R_{i} x_{3}} \varphi_{x}\left(P_{i}^{\prime}, P_{-i}\right)=.6$ and $\sum_{x R_{i} x_{3}} \varphi_{x}\left(P_{i}^{\prime \prime}, P_{-i}\right)=.8$.

Therefore, in this example the domain is not tight, and the equivalence of strategyproofness and monotone strategyproofness fails. 
Example 2 Let $X=\left\{x_{1}, x_{2}, x_{3}, x_{4}\right\}$, and let $\mathcal{D}$ be the domain composed of the three preference orderings depicted in Table 3.

\begin{tabular}{ccc}
\hline$P_{i}$ & $P_{i}^{\prime}$ & $P_{i}^{\prime \prime}$ \\
\hline \hline$x_{1}$ & $x_{2}$ & $x_{3}$ \\
$x_{2}$ & $x_{3}$ & $x_{4}$ \\
$x_{3}$ & $x_{1}$ & $x_{2}$ \\
$x_{4}$ & $x_{4}$ & $x_{1}$ \\
\hline
\end{tabular}

Table 3: Another non-tight domain

Note that $K\left(P^{\prime}, P^{\prime \prime}\right)=\{(a, d),(b, c),(b, d)\}$, so the domain is not tight. If $\varphi$ is a strategyproof social choice function that is not monotone strategyproof we need one of the following inequalities to be violated,

$$
\begin{aligned}
\pi_{x_{1}}^{\prime} & \geq \pi_{x_{1}}^{\prime \prime} \\
\pi_{x_{1}}^{\prime}+\pi_{x_{2}}^{\prime} & \geq \pi_{x_{1}}^{\prime \prime}+\pi_{x_{2}}^{\prime \prime} \\
\pi_{x_{1}}^{\prime}+\pi_{x_{2}}^{\prime}+\pi_{x_{3}}^{\prime} & \geq \pi_{x_{1}}^{\prime \prime}+\pi_{x_{2}}^{\prime \prime}+\pi_{x_{3}}^{\prime \prime}
\end{aligned}
$$

From strategyproofness we have

$$
\begin{aligned}
& P^{\prime \prime} \gg^{P^{\prime \prime}} P^{\prime} \quad \Rightarrow \quad \pi_{x_{3}}^{\prime \prime}+\pi_{x_{4}}^{\prime \prime}+\pi_{x_{2}}^{\prime \prime} \geq \pi_{x_{3}}^{\prime}+\pi_{x_{4}}^{\prime}+\pi_{x_{2}}^{\prime} \quad \Leftrightarrow \quad \pi_{x_{1}}^{\prime \prime} \leq \pi_{x_{1}}^{\prime} \\
& P^{\prime \prime} \gg^{P^{\prime \prime}} P^{\prime} \quad \Rightarrow \quad \pi_{x_{3}}^{\prime \prime}+\pi_{x_{4}}^{\prime \prime \prime} \geq \pi_{x_{3}}^{\prime}+\pi_{x_{4}}^{\prime} \quad \Leftrightarrow \quad \pi_{x_{1}}^{\prime \prime}+\pi_{x_{2}}^{\prime \prime} \leq \pi_{x_{1}}^{\prime}+\pi_{x_{2}}^{\prime}
\end{aligned}
$$

and

$$
P^{\prime} \gg^{P^{\prime}} P^{\prime \prime} \quad \Rightarrow \quad \pi_{x_{2}}^{\prime}+\pi_{x_{3}}^{\prime}+\pi_{x_{1}}^{\prime} \geq \pi_{x_{2}}^{\prime \prime}+\pi_{x_{3}}^{\prime \prime}+\pi_{x_{1}}^{\prime \prime}
$$

Eqs. (15), (16) and (17) imply that (12), (13) and (14) must hold, respectively. So we must have $P^{\prime} \gg^{P} P^{\prime \prime}$.

Therefore, a tight domain is not necessary to obtain the equivalence between strategyproofness and monotone strategyproofness. 


\section{Discussion}

\subsection{Weak preferences}

Until now we have only considered the case of strict preference domains. In this section we discuss how our results extend to the case of weak preferences. A weak preference relation $R_{i}$ for agent $i$ over $X$ is a complete, reflexive and transitive binary relation on $X$. Given a preference relation $R_{i}$ we denote by $P_{i}$ and $I_{i}$ the corresponding strict and indifference preference relation, respectively. That is, $x P_{i} x^{\prime}$ if $x R_{i} x^{\prime}$ and not $x^{\prime} R_{i} x$, and $x I_{i} x^{\prime}$ if both $x R_{i} x^{\prime}$ and $x^{\prime} R_{i} x$ hold. We denote by $\mathcal{R}$ the domain of all possible preference profiles over $X$.

The natural extension of the Kemeny set concept for weak preference relations is the notion of intermediate preferences introduced by Grandmont (1978).

Definition $4 R_{i}^{\prime}$ is between $R_{i}$ and $R_{i}^{\prime \prime}$ (noted as $R_{i}^{\prime} \in\left(R_{i}, R_{i}^{\prime \prime}\right)$ ) if for all $x, x^{\prime} \in X$,

(a) $x R_{i} x^{\prime}$ and $x R_{i}^{\prime \prime} x^{\prime}$ imply $x R_{i}^{\prime} x^{\prime}$.

(b) $x P_{i} x^{\prime}$ and $x P_{i}^{\prime \prime} x^{\prime}$ imply $x P_{i}^{\prime} x^{\prime}$.

(c) $\left(x I_{i} x^{\prime}\right.$ and $\left.x P_{i}^{\prime \prime} x^{\prime}\right)$ or $\left(x P_{i} x^{\prime}\right.$ and $\left.x I_{i}^{\prime \prime} x^{\prime}\right)$ imply $x R_{i}^{\prime} x^{\prime}$.

One easily see that for a triple $\left(P_{i}, P_{i}^{\prime}, P_{i}^{\prime \prime}\right)$ of strict preferences $K\left(P_{i}^{\prime}, P_{i}\right) \subseteq K\left(P_{i}^{\prime \prime}, P_{i}\right)$ implies that condition (b) of Definition 4 holds. In this case a natural definition of monotone strategyproofness would be that for any triple of preference relations $\left(R_{i}, R_{i}^{\prime}, R_{i}^{\prime \prime}\right)$ such that $R_{i}^{\prime} \in\left(R_{i}, R_{i}^{\prime \prime}\right)$, it holds that $R_{i}^{\prime}$ dominates $R_{i}^{\prime \prime}$ with respect to $R_{i}$. One could then expect that monotone strategyproofness would be equivalent to strategyproofness in this setting. However, there exist situations where this property does not hold. To see this, let $R_{i}^{\prime} \in$ $\left(R_{i}, R_{i}^{\prime \prime}\right)$ such that for some preference profile $R_{-i}$ we have $\varphi\left(R_{i}^{\prime}, R_{-i}\right)=x^{\prime}$ and $\varphi\left(R_{i}^{\prime \prime}, R_{-i}\right)=$ $x^{\prime \prime}$, and assume that $x^{\prime} \neq x^{\prime \prime}$. Suppose that we have $x^{\prime \prime} P_{i} x^{\prime}, x^{\prime} I_{i}^{\prime} x^{\prime \prime}$ and $x^{\prime} I_{i}^{\prime \prime} x^{\prime \prime}$. That is, for the pair $\left(x^{\prime}, x^{\prime \prime}\right)$ we have to consider the second part of condition (c) of Definition 4 . It is then easy to see that the pair $\left(x^{\prime}, x^{\prime \prime}\right)$ does not violate the fact that $R_{i}^{\prime}$ is between $R_{i}$ and $R_{i}^{\prime \prime}$, yet we clearly have that $R_{i}^{\prime}$ cannot dominate $R_{i}^{\prime \prime}$ with respect to $R_{i}$.

In other words, the equivalence between strategyproofness and monotone strategyproofness is not assured when considering indifferences. The reason behind is that when an agent is indifferent between two alternatives, strategyproofness does not impose any particular selection among these two alternatives. That is, we may well have that two alternatives, say, $x^{\prime}$ 
and $x^{\prime \prime}$ are indifferent under two preferences $R_{i}^{\prime}$ and $R_{i}^{\prime \prime}$ yet $x^{\prime}$ is strictly preferred to $x^{\prime \prime}$ under $R_{i}$. Strategyproofness in this case does not impose that alternative $x^{\prime}$ should be chosen over $x^{\prime \prime}$ under either $R_{i}^{\prime}$ or $R_{i}^{\prime \prime}$ for some preference profile $P_{-i}$.

\subsection{Comparing preferences}

Theorems 1 and 2 show that Kemeny set inclusion captures dominance relations between different strategies in a strategyproof mechanism. One natural question to address is whether the converse also holds, i.e., if a preference ordering $P_{i}^{\prime}$ dominates another ordering $P_{i}^{\prime \prime}$ it is necessarily the case that $K\left(P_{i}^{\prime}, P_{i}\right)$ is a subset of $K\left(P_{i}^{\prime \prime}, P_{i}\right)$. Note that since the starting point is the dominance relation between $P_{i}^{\prime}$ and $P_{i}^{\prime \prime}$ we can only infer some information about their Kemeny sets with the alternatives that can obtain with either $P_{i}^{\prime}$ or $P_{i}^{\prime \prime}$. That is, when comparing to preference orderings $P_{i}^{\prime}$ and $P_{i}^{\prime \prime}$ we are limited to the set of pairs of alternatives that can be realized for some profile $P_{-i}$, i.e., the pairs $\left(x, x^{\prime}\right)$, such that $\varphi\left(P_{i}^{\prime}, P_{-i}\right)=x$ and $\varphi\left(P_{i}^{\prime \prime}, P_{-i}\right)=x^{\prime}$. We accordingly refine the concept of the Kemeny set by restricting comparisons to be done only on such sets of pairs of alternatives.

The joint range of two preference orderings $P_{i}$ and $P_{i}^{\prime}$ is the set of pairs alternatives $\left(v, v^{\prime}\right)$ for which there exists a profile $P_{-i}$ such that $\varphi\left(P_{i}, P_{-i}\right)=v$ and $\varphi\left(P_{i}^{\prime}, P_{-i}\right)=v^{\prime}$. The joint range of $P_{i}$ and $P_{i}^{\prime}$ is denoted $J\left(P_{i}, P_{i}^{\prime}\right)$ and $J$ when there is no possible confusion.

Definition 5 Given three preference orderings $P_{i}, P_{i}^{\prime}$ and $P_{i}^{\prime \prime}$, the Kemeny set of $P^{\prime}$ with respect to $P$ on joint range with $P^{\prime \prime}$ is the set of all pairs $\left(x, x^{\prime}\right) \in X \times X$ that are not ordered identically in $P_{i}$ and $P_{i}^{\prime}$ and that belong to the joint range of $P_{i}^{\prime}$ and $P_{i}^{\prime \prime}$, i.e.,

$$
\widehat{K}\left(P_{i}^{\prime}, P_{i}, P_{i}^{\prime \prime}\right) \equiv\left\{\left(x, x^{\prime}\right) \in J\left(P_{i}^{\prime}, P_{i}^{\prime \prime}\right): x^{\prime} P_{i} x \text { and } x P_{i}^{\prime} x^{\prime}\right\}
$$

Proposition 2 Let $\varphi$ be a deterministic strategyproof social choice function. If $P_{i}^{\prime}$ dominates $P_{i}^{\prime \prime}$ with respect to $P_{i}$, then $\widehat{K}\left(P_{i}^{\prime}, P_{i}, P_{i}^{\prime \prime}\right) \subseteq \widehat{K}\left(P_{i}^{\prime \prime}, P_{i}, P_{i}^{\prime}\right)$.

Proof Let $P_{i}, P_{i}^{\prime}$ and $P_{i}^{\prime \prime}$ such that $P_{i}^{\prime}$ dominates $P_{i}^{\prime \prime}$ with respect to $P_{i}$, and let $P_{-i}$ such that $\varphi\left(P_{i}^{\prime}, P_{-i}\right) \neq \varphi\left(P_{i}^{\prime \prime}, P_{-i}\right)$. Define $x=\varphi\left(P_{i}^{\prime}, P_{-i}\right)$ and $x^{\prime}=\varphi\left(P_{i}^{\prime \prime}, P_{-i}\right)$. So, $\left(x, x^{\prime}\right) \in$ $J\left(P_{i}^{\prime}, P_{i}^{\prime \prime}\right)$ and since $P_{i}^{\prime}$ dominates $P_{i}^{\prime \prime}$ with respect to $P_{i}, x P_{i} x^{\prime}$. It is sufficient to show

$$
\left(x, x^{\prime}\right) \in \widehat{K}\left(P_{i}^{\prime}, P_{i}^{\prime \prime}, P_{i}\right) \quad \Rightarrow \quad\left(x, x^{\prime}\right) \in \widehat{K}\left(P_{i}^{\prime \prime}, P_{i}^{\prime}, P_{i}\right)
$$


Suppose then that $\left(x, x^{\prime}\right) \in \widehat{K}\left(P_{i}^{\prime}, P_{i}, P_{i}^{\prime \prime}\right)$ and $\left(x, x^{\prime}\right) \notin \widehat{K}\left(P_{i}^{\prime \prime}, P_{i}, P_{i}^{\prime}\right)$. So $x P_{i}^{\prime \prime} x^{\prime}$. This is tantamount to $\varphi\left(P_{i}^{\prime}, P_{-i}\right) P_{i}^{\prime \prime} \varphi\left(P_{i}^{\prime \prime}, P_{-i}\right)$, which contradicts strategyproofness. So, $x^{\prime} P_{i}^{\prime \prime} x$, i.e., $\left(x, x^{\prime}\right) \in \widehat{K}\left(P_{i}^{\prime \prime}, P_{i}, P_{i}^{\prime}\right)$.

Example 3 To see the role that the joint support plays in the result of Proposition 2, consider the standard median voter social choice function with the single-peaked preference domain. It is well known that for the single-peaked domain the median voter rule is strategyproof (Moulin, 1980).

Single peaked-preferences are defined as follows. There exists an ordering $L$ of the alternatives. Without loss of generality assume that the ordering is $x_{1}, \ldots, x_{\ell}$, i.e., $x_{h} L x_{h^{\prime}}$ for each $h<h^{\prime} \leq \ell$. Agents having single-peaked preferences means that for each agent $i \in N$ there is an alternative $x_{k}$ such that for each $h, h^{\prime}$ where $h<h^{\prime} \leq k$ (resp. $k \geq h>h^{\prime}$ ), then we have $x_{k} R_{i} x_{h} P_{i} x_{h^{\prime}}{ }^{14}$ For simplicity, assume here that there is an odd number of agents.

Let $i$ be an agent and $P_{i}$ his true preferences where $x$ is the most preferred alternative (the peak) according to $P_{i}$. Consider now two preference orderings (that are single-peaked with the order $L$ ), $P_{i}^{\prime}$ and $P_{i}^{\prime \prime}$, where $x^{\prime}$ and $x^{\prime \prime}$ are their respective peaks. Suppose that $x L x^{\prime} L x^{\prime \prime}$, i.e., the peak of $P_{i}^{\prime}$ lies between that of $P_{i}$ and $P_{i}^{\prime \prime}$. It is not difficult to see that for any profile $P_{-i}$ of the other agents, the individual with preferences $P_{i}$ prefers the outcome with $\left(P_{i}^{\prime}, P_{-i}\right)$ than the outcome with $\left(P_{i}^{\prime \prime}, P_{-i}\right)$. That is, $P_{i}^{\prime}$ dominates $P_{i}^{\prime \prime}$. Yet, suppose that there exists a pair of alternatives, say $x_{1}$ and $x_{2}$, such that $\left(x_{1}, x_{2}\right) \in K\left(P_{i}^{\prime}, P_{i}\right)$ yet $\left(x_{1}, x_{2}\right) \notin K\left(P_{i}^{\prime \prime}, P_{i}\right)$. We claim that $\left(x_{1}, x_{2}\right) \notin J\left(P_{i}^{\prime}, P_{i}^{\prime \prime}\right)$.

To see this, without loss of generality suppose that $\varphi\left(P_{i}^{\prime}, P_{-i}\right)=x_{1}$ and $\varphi\left(P_{i}^{\prime \prime}, P_{-i}\right)=x_{2}$. first that $x_{1} L x^{\prime}$. If $x_{1} L x^{\prime}$, then by choosing $P_{i}^{\prime \prime}$ instead of $P_{i}^{\prime}$ agent $i$ cannot change the outcome, so $x_{1}=x_{2}$, a contradiction. So, $x^{\prime} L x_{1}$. Using symmetric argument we obtain $x_{2} L x^{\prime \prime}$. So we have $x L x^{\prime} L x_{1} L x_{2} L x^{\prime \prime}$, which contradicts $\left(x_{1}, x_{2}\right) \in K\left(P_{i}^{\prime}, P_{i}\right)$. If $x_{2} L x_{1}$, a similar arguments leads to a contradiction, too. It is important to note that $\left(x_{1}, x_{2}\right) \in$ $K\left(P_{i}^{\prime}, P_{i}\right)$ and $\left(x_{1}, x_{2}\right) \notin K\left(P_{i}^{\prime \prime}, P_{i}\right)$ does not contradict single-peakedness. So, even though $P_{i}^{\prime}$ dominates $P_{i}^{\prime \prime}$ we can still have $K\left(P_{i}^{\prime}, P_{i}\right) \nsubseteq K\left(P_{i}^{\prime \prime}, P_{i}\right)$. However, it cannot be that $\widehat{K}\left(P_{i}^{\prime}, P_{i}^{\prime \prime}, P_{i}\right) \nsubseteq \widehat{K}\left(P_{i}^{\prime \prime}, P_{i}^{\prime}, P_{i}\right)$.

The problem becomes more delicate when we consider non-deterministic social choice

\footnotetext{
${ }^{14}$ See Ballester and Haeringer (2011) for a characterization of the single-peaked domain.
} 
functions. Consider the following situation with three alternatives,

\begin{tabular}{ccc}
$P_{i}$ & $P_{i}^{\prime}$ & $P_{i}^{\prime \prime}$ \\
\hline$x_{1}$ & $x_{2}$ & $x_{1}$ \\
$x_{2}$ & $x_{1}$ & $x_{3}$ \\
$x_{3}$ & $x_{3}$ & $x_{2}$
\end{tabular}

Suppose that we know that $P_{i}^{\prime} \gg^{P_{i}} P_{i}^{\prime \prime}$. Clearly, $K\left(P_{i}^{\prime}, P_{i}\right) \nsubseteq K\left(P_{i}^{\prime \prime}, P_{i}\right)$. Consider any profile $P_{-i}$ and let $\pi_{x}^{\prime}=\varphi_{x}\left(P_{i}^{\prime}, P_{-i}\right)$ and $\pi_{x}^{\prime \prime}=\varphi_{x}\left(P_{i}^{\prime \prime}, P_{-i}\right)$. Assuming that $\varphi$ is strategyproof we obtain $\pi_{x_{1}}^{\prime} \geq \pi_{x_{1}}^{\prime}$ and $\pi_{x_{1}}^{\prime} \leq \pi_{x_{1}}^{\prime \prime}$, where the first and second inequalities comes from $P_{i}^{\prime} \gg^{P_{i}} P_{i}^{\prime \prime}$ and $P_{i}^{\prime \prime} \gg^{\prime \prime} P_{i}^{\prime}$, respectively. So, $\pi_{x_{1}}^{\prime}=\pi_{x_{1}}^{\prime \prime}$. Note that it could be that the probability to obtain alternative $x_{1}$ may depend on the profile $P_{-i}$, but the playing $P_{i}^{\prime}$ or $P_{i}^{\prime \prime}$ cannot affect it. One may be tempted then to exclude alternative $x_{1}$ from the set of alternatives considered when comparing $P_{i}^{\prime}$ and $P_{i}^{\prime \prime}$. Indeed, by doing so we see that $P_{i}^{\prime}$ does not reverse the relative ranking of alternatives $x_{2}$ and $x_{3}$ while $P_{i}^{\prime \prime}$ does.

However, as the next example shows, ignoring alternatives whose probability does not depend on the preference ordering chosen by the individual does not solve our problem. To see this, consider a slightly modified example, where the numbers in parenthesis are the probabilities that the corresponding alternative obtains for any profile $P_{-i}$ (it is readily verified that in case $\varphi$ is strategyproof),

\begin{tabular}{ccc}
$P_{i}$ & $P_{i}^{\prime}$ & $P_{i}^{\prime \prime}$ \\
\hline$x_{1}(.5)$ & $x_{2}(.5)$ & $x_{3}(.5)$ \\
$x_{2}(.3)$ & $x_{1}(.3)$ & $x_{1}(.2)$ \\
$x_{3}(.2)$ & $x_{3}(.2)$ & $x_{2}(.3)$
\end{tabular}

When comparing $P_{i}^{\prime}$ and $P_{i}^{\prime \prime}$ no alternatives can be discarded, yet we clearly have $K\left(P_{i}^{\prime}, P_{i}\right) \nsubseteq$ $K\left(P_{i}^{\prime \prime}, P_{i}\right)$.

\subsection{Cardinal environments}

The concept of monotone strategyproofness can be easily adapted to cardinal environments, i.e., when an agent is characterized by a utility vector over the set of alternatives and agents have expected utility preferences over lotteries. A few more definitions are needed before going further: 
A type space is a non-empty subset of $\times_{i \in N} \mathbb{R}^{|X|}$, and an agent's type is a vector in $\mathbb{R}^{|X|}$. Given a type space $T$, a mechanism is a mapping $\varphi: T \rightarrow \Delta(X)$. We denote by $u_{i}$ a generic type of agent $i$, and $u=\left(u_{i}\right)_{i \in N}$ is a type profile. Given a (true) type profile $u$ and a reported type profile $u^{\prime}$, the expected utility of agent $i$ is given by the inner product $u_{i} \cdot \varphi(u)$. A mechanism is incentive compatible on a type space $T$ if, for each $i \in N$, for each $u \in T$ and each $u^{\prime} \in T$ such that $u_{-i}=u_{-i}^{\prime}$, we have $u_{i} \cdot\left(\varphi\left(u_{i}, u_{-i}\right)-\varphi\left(u_{i}^{\prime}, u_{-i}\right)\right) \geq 0$.

Definition 6 A mechanism is monotone incentive compatible on a type space $T$ if, for each $i \in N$, for each $u \in T$ and each $u^{\prime}, u^{\prime \prime} \in T$ such that $u_{-i}=u_{-i}^{\prime}=u_{-i}^{\prime \prime}$ and $u_{i}^{\prime}=\alpha \cdot u_{i}+(1-\alpha) \cdot u_{i}^{\prime \prime}$ for some $\alpha \in(0,1)$, we have $u_{i} \cdot\left(\varphi\left(u_{i}^{\prime}, u_{-i}\right)-\varphi\left(u_{i}^{\prime \prime}, u_{-i}\right)\right) \geq 0$.

We can now introduce the counterpart of Proposition 1 for cardinal mechanisms. ${ }^{15}$

Proposition 3 A mechanism is incentive compatible if, and only if, it is monotone incentive compatible.

Proof That a monotone incentive compatible mechanism is also incentive compatible is obvious. Consider then an incentive compatible mechanism $\varphi$ on a type space $T$. Let $u_{i}$ be any admissible type for agent $i$, and $u_{i}^{\prime}$, $u_{i}^{\prime \prime}$ such that $u_{i}^{\prime}=(1-\alpha) \cdot u_{i}+\alpha \cdot u_{i}^{\prime \prime}$ for some $\alpha \in(0,1)$. Since $\varphi$ is incentive compatible,

$$
\begin{aligned}
& u_{i}^{\prime} \cdot\left(\varphi\left(u_{i}^{\prime}, u_{-i}\right)-\varphi\left(u_{i}^{\prime \prime}, u_{-i}\right)\right) \geq 0 \\
& u_{i}^{\prime \prime} \cdot\left(\varphi\left(u_{i}^{\prime \prime}, u_{-i}\right)-\varphi\left(u_{i}^{\prime}, u_{-i}\right)\right) \geq 0
\end{aligned}
$$

Multiplying the second constraint by $\alpha$ and adding up the two inequalities and rearranging yields

$$
\left(u_{i}^{\prime}-\alpha u_{i}^{\prime \prime}\right) \cdot\left(\varphi\left(u_{i}^{\prime}, u_{-i}\right)-\varphi\left(u_{i}^{\prime \prime}, u_{-i}\right)\right) \geq 0
$$

Note that $u_{i}^{\prime}-\alpha u_{i}^{\prime \prime}=(1-\alpha) \cdot u_{i}$. Since $\alpha \in(0,1)$ we obtain $u_{i} \cdot\left(\varphi\left(u_{i}^{\prime}, u_{-i}\right)-\varphi\left(u_{i}^{\prime \prime}, u_{-i}\right)\right) \geq 0$.

A straightforward application of Proposition 3 is for incentive compatible auction mechanisms with private values. Consider the case when agents' types are real numbers (their value of the good to be auctioned). Our result then simply says that if an agent's private value for the auctioned good is, say, $x$, then bidding $x^{\prime}<x$ dominates bidding $x^{\prime \prime}<x^{\prime}$.

\footnotetext{
${ }^{15}$ The proof of Proposition 3 is built on the proof of Proposition 1 in Carroll (2012).
} 


\section{Conclusions}

We show that under strategyproof mechanism one can meaningfully compare the extent of preference misrepresentation by comparing pairs of alternatives. We define the concept of monotone strategyproofness, which captures the link between incentives and the extent of a misrepresentation: a larger extend of misrepresentation makes the agent (weakly) worse off. Remarkably, requiring monotone strategyproofness does not reduce the set of strategyproof social choice functions. This result shows that imposing strategyproofness (or incentive compatibility) does not only consist of imposing the existence of one dominating strategy (the one corresponding to the true type) but imposes the existence of a large collection of dominance relations between strategies, thereby providing further evidence that strategyproofness is a very demanding property.

Our results also shed light on the complications that arise when social choice functions are non-deterministic, or are defined on domains with indifferences. Strategyproofness in the non-deterministic case imposes that the truthful strategy stochastically dominates any other strategy. It is well known that the mere existence of a stochastically dominating strategy can be very challenging in a general setting, so it is not a surprise that one should impose some constraints on the domain to obtain the equivalence between strategyproofness and monotone strategyproofness for stochastic mechanisms. As for the case of domains with weak preferences we encounter stronger hurdles. Our discussion in Section 4.1 suggests indeed that obtaining a similar result for the case of weak preferences seems beyond reach. 


\section{REFERENCES}

[1] Andreoni, J. (1995) "Cooperation in public-goods experiments: Kindness or confusion?," American Economic Review, 85, 891-904.

[2] Ballester, M. A. and G. Haeringer (2011) "A Characterization of the Single-Peaked domain," Social Choice and Welfare, 36, 305-322.

[3] Barberà, S. (2011) "Strategyproof social choice," in K. J. Arrow, A. Sen and K. Suzumura (eds.), Handbook of Social Choice and Welfare, Volume 2, Elsevier: Amsterdam, Chapter 25, 731-831.

[4] Calsamiglia, C., G. Haeringer and F. Klijn (2010) "Constrained School Choice: An Experimental Study," American Economic Review, 100, 1860-1874.

[5] Carroll, G. (2012) "When are Local Incentive Constraints Sufficient?" Econometrica, 80, 661-686.

[6] Cason, T. N., T. Saijo, T. Sjöström and T. Yamato (2008), "Secure Implementation Experiments: Do Strategy-proof Mechanisms Really Work?," Games and Economic Behavior, 57, 206-235.

[7] Chatterji, S., R. Sanver and A. Sen (2013), "On Domains that Admit Well-Behaved Strategy-Proof Social Choice Functions," Journal of Economic Theory, 148, 1050-1073.

[8] Chen, Y. (2008) "Incentive-compatible Mechanisms for Pure Public Goods: A Survey of Experimental Research ," Handbook of Experimental Economics Results, C. R. Plott and V. Smith (Eds), 1, 625-643.

[9] Chen, Y. and T. Sönmez (2006) "School Choice: An Experimental Study," Journal of Economic Theory, 127, 202-231.

[10] Cho, W. J (2012) "Probabilistic assignment: a two-fold axiomatic approach," mimeo.

[11] Gibbard, A. (1973) "Manipulation of Voting Schemes: A General Result," Econometrica, 41, 587-601.

[12] Grandmont, J. M. (1978) "Intermediate Preferences and the Majority Rule Result," Econometrica, 46, 317-330. 
[13] Jackson, M. O. (1992) "Incentive Compatibility and Competitive Allocations," Economics Letters, 40, 299-302.

[14] Kagel, J. H. and D. Levin (1986). "The Winner's Curse and Public Information in Common Value Auctions," American Economic Review, 76, 894-920.

[15] Kemeny J. G. (1959) "Mathematics without numbers," Daedalus, 4, 577-591.

[16] Moulin, H. (1980) "On strategyproofness and single peakedness," Public Choice, 4, 437355.

[17] Palacios-Huerta, I. and O. Volij (2009) "Field Centipedes," American Economic Review. 99, 1619-1635.

[18] Pathak, P. A. and T. Sönmez (2008) "School Admissions Reform in Chicago and England: Comparing Mechanisms by Their Vulnerability to Manipulation," American Economic Review, forthcoming.

[19] Roth, A.E. (1982) "The Economics of Matching: Stability and Efficiency," Mathematics of Operations Research, 92, 617-628.

[20] Sato, S. (2013), "A Sufficient Condition for the Equivalence of strategyproofness and Non-manipulability by Preferences Adjacent to the Sincere One," Journal of Economic Theory, 148, 259-278.

[21] Satterthwaite, M.A. (1975), "Strategyproofness and Arrow's conditions: Existence and correspondence theorems for voting procedures and social welfare functions," Journal of Economic Theory, 10, 187-217. 